\title{
REPRESENTATION THEORY FOR DENUMERABLE MARKOV CHAINS $\left(^{1}\right)$
}

\author{
BY \\ JOHN G. KEMENY $\left({ }^{2}\right)$
}

1. Introduction. While there is an extensive theory of denumerable Markov chains, there is one major gap. If $P$ is the transition matrix, it has rarely been possible to compute $P^{n}$, the $n$-step transition probabilities, in any practical manner. By a representation theory for denumerable Markov chains we will mean a theory aimed at expressing $P$ in a form from which $P^{n}$, and quantities depending on $P^{n}$, can be easily computed. This paper presents a first step in the direction of such a theory.

If $P$ is a finite Markov chain transition matrix, then various canonical forms are available for the representation. They take the form

$$
P=Q S R
$$

where $Q=R^{-1}$, and hence

$$
P^{n}=Q S^{n} R
$$

If in addition $S$ is of a special form, where $S^{n}$ is easy to compute, the goal is achieved.

Our theory will develop a representation in the form (1.1), which is suitable for row-finite denumerable Markov chains (i.e., chains for which each row of $P$ has only a finite number of nonzero entries). By duality, we also obtain a representation for column-finite chains.

There is an apparent difficulty in this: Since our matrices are infinite, they are not, in general, associative, nor is it clear what we mean by an inverse of a matrix. However, as long as $Q, R$, and $S$ are row-finite, each entry on the right of (1.2) is a finite sum, and hence there are no difficulties. We further make sure that the condition of $Q$ being an inverse of $R$ depends only on finite submatrices of each matrix. Thus we can reduce the existence and uniqueness of inverses to the same question for finite matrices.

The present paper works out the representation for a very large class of chains, which we named the slowly spreading chains $\left({ }^{3}\right)$ (see $\S 2$ ). For these chains $Q$ and $R$

Received by the editors November 12, 1965.

( $\left.{ }^{1}\right)$ Presented to the Institute of Mathematical Statistics in September 1965.

$\left({ }^{2}\right)$ The preparation of this paper was supported by the National Science Foundation.

$\left({ }^{3}\right)$ Various generalizations of this representation are presented in $\$ 8$. 
turn out to be triangular, by which we will mean that they are positive on the main diagonal and zero above the diagonal. For such matrices it is easily seen that $Q R=I$ or $R Q=I$ simply states that finite submatrices consisting of the first $n$ rows and columns be inverses of each other in the ordinary sense. And since the determinant of such a finite triangular matrix is simply the product of the (positive) diagonal entries, we see that every triangular matrix has a unique inverse, which is again triangular.

The matrix $S$ will be the matrix having ones just to the right of the main diagonal, i.e., $S_{i, i+1}=1$ with all other entries 0 . This is the transition matrix of the chain which always takes one step to the right, and hence $S^{n}$ simply has a one $n$ places to the right of the main diagonal in each row.

The terminology and notation of the paper is taken from [5]. We note the following often used symbols: 1 is the column vector of all ones, so that $W 1$ gives the row-sums of the matrix $W .{ }^{k} N_{i j}$ is the mean number of visits to $j$, starting at $i$, before $k$ is reached.

The author is indebted to the Karlin and McGregor representation of random walks [1], of which the present theory is both a simplification and an extension. The exact connection will be shown in $\S 6$.

2. Slowly spreading chains. A slowly spreading chain (s.s.ch.) has the natural numbers as states and it cannot move more than one step to the right. That is $P_{i j}=0$ if $j>i+1$. To assure that the states communicate, we require that $p_{i}=$ $P_{i-1, i}>0$ for all $i$, but that $p_{i}<1$ infinitely often; and that state 0 can be reached from every state. This is a very rich family of Markov chains, including some classes of chains that have received detailed study in the literature.

To obtain the matrix $R$ of the representation, we proceed as follows: Let the 0 th row of $R$ be the vector $(1,0,0, \ldots)$. And let the $(n+1)$ st row be the $n$th row times $P$. Thus multiplication by $P$ has the same effect as shifting the rows of $R$ up by one. Or in matrix language,

$$
R P=S R
$$

We note that $R_{n j}=P_{0 j}^{(n)}$. Thus if $P$ has row-sums equal to 1 (the chain cannot stop) then $R$ has the same property. Also $R$ is nonnegative. We verify, by induction, that $R$ is triangular. Here is where we make essential use of the fact that $P$ is a s.s.ch.

Thus $R$ has a unique inverse $Q$, which is again triangular, and it has row-sums equal to 1 if $R$ does. But $Q$ is not nonnegative.

From (2.1) we obtain, by induction, that

$$
R P^{n}=S^{n} R,
$$

and when we multiply both sides of the equation, on the left, by $Q$, and use the fact that all the matrices are row-finite,

$$
P^{n}=Q S^{n} R \text {. }
$$


Thus we have obtained a useful representation of the $n$th power of $P$ (hence of the $n$-step transition probabilities). It is important to note that every entry on the right is a finite sum.

It is sometimes useful to know that we can start the representation by finding $Q$. The matrix $R$ was determined by specifying its first row, and by (2.1). Similarly, $Q$ satisfies the equation

$$
P Q=Q S
$$

This equation together with the requirement that $Q$ be triangular, and $Q_{00}=1$, will determine $Q$. Then we obtain $R$ as the unique inverse of $Q$.

Let us introduce three matrices consisting of 0's and 1's. $E$ is the matrix of all 1's.

$$
T^{(n)}=\sum_{k=0}^{n} S^{k}, \quad T^{*}=\lim _{n} T^{(n)}, \quad T=E-T^{*}
$$

Then $T^{(n)}$ has ones on the main diagonal, and $n$ places to the right, $T^{*}$ has ones on the main diagonal and above, while $T$ has ones below the main diagonal.

If $N_{i j}^{(n)}$ is the mean number of times from $i$ to $j$ in $n$ steps, it follows immediately from (2.2) that

$$
N^{(n)}=Q T^{(n)} R
$$

3. The interpretation of $Q$. An interpretation of $R$ resulted directly from its construction, but the interpretation of $Q$ is not obvious. Let us introduce the finite matrices ${ }^{i} P$ ( $P$ before $i$ ), consisting of rows and columns $0,1, \ldots, i-1$ of $P$. It represents the behavior of the chain started in one of the first $i$ states, before state $i$ is reached. It may be thought of as a finite transient chain in its own right.

Then we claim that the $i$ th row of $Q$ is proportional to the coefficients of the characteristic equation of ${ }^{i} P$. More precisely, that the characteristic equation of ${ }^{i} P$ is

$$
\chi(x)=\beta_{i} \sum_{n=0}^{i} Q_{i n} x^{n}
$$

Since $Q$ is determined by the fact that it is the unique inverse of $R$, its $i$ th row is determined by the fact that multiplied into columns $0,1, \ldots, i-i$ of $R$ it yields 0 , and on column $i$ it yields 1 . Suppose that

$$
\chi(x)=\sum_{n=0}^{i} c_{n} x^{n}
$$


Since $R_{n j}=P_{0 j}^{(n)}$, the sum $\sum c_{n} R_{n j}$ is the $0-j$ entry of the matrix sum $\chi(P)$. As long as $j<i$ and $n \leqq i$, this is the same as the $0-j$ entry of $\chi\left({ }^{i} P\right)=0$, since if $j$ is reached in no more than $i$ steps from 0 , the chain cannot have reached $i$ previously. Thus $\left(c_{0}, c_{1}, \ldots, c_{i}\right)$ multiplied into columns $0,1, \ldots, i-1$ of $R$ yields 0 . And multiplied into the $i$ th column we obtain the single term $c_{i} P_{0 i}^{(i)}=1 \cdot \beta_{i}$. Hence $Q_{i n}=c_{n} / \beta_{i}$, as was to be shown.

We could have started with this interpretation of $Q$, found $R$ from the above argument, and then argued that $Q S^{k} R=Q R P^{k}$ from the interpretation of $R$, hence $Q S^{k} R=P^{k}$.

We also obtain an interpretation for the sum

$$
{ }^{i} N=\sum_{n=0}^{i}(Q T)_{i n}\left({ }^{i} P\right)^{n}
$$

by evaluating

$$
\begin{aligned}
{ }^{i} N\left(I-{ }^{i} P\right) & =\sum_{n=0}^{i}\left(\sum_{k=n+1}^{i} Q_{i k}\right)\left(\left({ }^{i} P\right)^{n}-\left({ }^{i} P\right)^{n+1}\right) \\
& =\sum_{k=0}^{i} Q_{i k} \sum_{n=0}^{k-1}\left(\left({ }^{i} P\right)^{n}-\left({ }^{i} P\right)^{n+1}\right) \\
& =\sum_{k=0}^{i} Q_{i k}\left(I-\left({ }^{i} P\right)^{k}\right) \\
& =I .
\end{aligned}
$$

Thus ${ }^{i} N=\left(I-{ }^{i} P\right)^{-1}$ is the fundamental matrix of the transient chain ${ }^{i} P$. And by taking the $0-j$ entry of ${ }^{i} P$ in (3.2) we obtain

$$
(Q T R)_{i j}={ }^{i} N_{0 j}
$$

for any $j<i$. This quantity is finite for all chains whose states communicate. Incidentally, the equation (3.3) holds even if $j \geqq i$, since both sides are 0 in this case.

Since (3.3) will play a key role in evaluating various interesting quantities, it should be pointed out that in the matrix product $Q T R$ each entry is the sum of only a finite number of nonzero terms. Thus, once either $Q$ or $R$ is known, a given entry in $Q T R$ can be found in a finite number of steps. It happens to be a procedure ideally suited for machine computation, which has been most helpful in computing examples.

We can go one step further. Since a s.s.ch. cannot "jump over" a state, we know that if $j$ and $k$ are both less than $i$, then ${ }^{i} N_{k j}={ }^{i} N_{0 j}-{ }^{k} N_{0 j}$. Thus

$$
{ }^{i} N_{k j}=(Q T R)_{i j}-(Q T R)_{k j} \quad \text { for } j, k<i .
$$


The row-sums of $Q T$ are also interesting. We introduce

$$
m=Q T 1
$$

as the vector of row-sums. And from (3.3),

$$
m_{i}=(Q T 1)_{i}=(Q T R 1)_{i}=\sum_{j}{ }^{i} N_{0 j}=M_{0 i},
$$

the mean time from 0 to reach $i$. It is a special property of s.s.ch. that this quantity is always finite.

Thus, from the interpretation of $Q$ we obtained a number of quantities of interest for all s.s.ch.

4. Transient slowly spreading chains. We will next investigate the case of a transient s.s.ch. This is characterized by the fact that $N=\lim _{n} N^{(n)}$ is finite. This limit is easy to compute, thanks to special properties of our matrices:

$$
\begin{aligned}
N & =\lim _{n} Q T^{(n)} R & & \text { from }(2.6) \\
& =Q \lim _{n}\left[T^{(n)} R\right] & & \text { since } Q \text { is row-finite } \\
& =Q T^{*} R & & \text { by monotonicity. }
\end{aligned}
$$

Hence,

$$
N=Q T^{*} R
$$

This is finite if and only if $T^{*} R$ is finite, which requires that $R$ have finite-column sums. We could have deduced this condition more simply from the interpretation of $R$, since the $j$ th column sum of $R$ is $N_{0 j}$. But the fact that we can obtain all of $N$ as simply as (4.1) is a pleasant surprise.

We might as well point out that a s.s.ch. can be classified entirely from one column of $R$, say the 0 th. It must have a finite-column sum $\left(N_{00}\right)$ in the transient case. In the recurrent case the column sum is always infinite. For the chain to be null, we require in addition that $\lim _{n} R_{n 0}=0$. If the chain is ergodic, the column does not tend to 0 . If it is noncyclic it tends to a positive limit, while in the cyclic case it oscillates.

Let us briefly investigate the case where $P$ does not have all of its row-sums equal to 1 . Of course, such a chain can stop, and hence, with states communicating, it is transient. In this case the row-sums of $Q$ and $R$ are very interesting. Let $q=Q 1$ and $r=R 1$ be the vectors representing the row-sums. It follows immediately from the interpretation of $R$ that

$$
r_{n}=\sum_{j} P_{0 j}^{(n)}
$$

is the probability that the chain started at 0 does not stop in $n$ steps. 
It is only slightly more difficult to interpret $q$. It is finite valued, since $Q$ is rowfinite. Furthermore,

$$
P q=P Q 1=Q S 1=Q 1=q .
$$

Thus if we start the process at 0 , and stop it at $i$, then $q\left(X_{n}\right)$ is a martingale, and

$$
1=q_{0}=H_{0 i} q_{i}
$$

or $q_{i}=1 / H_{0 i}$, where $H_{0 i}$ is the probability of reaching $i$ from 0 .

Thus $q$ and $r$ are nonnegative, $q \geqq 1$ while $r \leqq 1$, and either vector equals 1 only if $P$ has row-sums equal to 1 . Furthermore, $q_{n}$ is monotone increasing to a limit $q_{\infty}$ (possibly $+\infty$ ), while $r_{n}$ is monotone decreasing to $r_{\infty}$. It is clear from the interpretation that

$$
r_{\infty}=1 / q_{\infty}=P r_{0}[\text { chain does not stop }]
$$

And since

$$
\operatorname{Pr}_{0}[\text { chain does not stop }]=H_{0 i} \cdot \operatorname{Pr}_{i}[\text { chain does not stop }]
$$

we obtain

$$
\operatorname{Pr}_{i}[\text { chain does not stop }]=q_{i} / q_{\infty} \text {. }
$$

5. Recurrent slowly spreading chains. For recurrent chains there is a positive vector $\alpha$ such that $\alpha P=\alpha$, and this is unique up to a constant multiple. For a noncyclic ergodic chain $P_{0 j}^{(n)}$ converges to $\alpha_{j}$, for the normalized version of $\alpha$. Hence the limits of the columns of $R$ determine $\alpha$. For a cyclic ergodic chain the limits of the nonzero entries in the various columns will serve.

However, for the null case we must rely on the ratio limit theorem:

$$
\lim _{n} \frac{N_{0 j}^{(n)}}{N_{0 i}^{(n)}}=\frac{\alpha_{j}}{\alpha_{i}}
$$

Thus we must take the sum of the first $n$ terms in column $j$ of $R$, and divide by the sum of the first $n$ terms in column $i$, and let $n$ tend to infinity. Since $\alpha$ is a fundamental quantity, we wish for a simpler method of obtaining $\alpha$. Unfortunately, we have only a partial result in this direction.

Suppose that $\alpha Q$ is well defined and finite valued (which need not be the case). Then

$$
\alpha Q=(\alpha P) Q=\alpha(P Q)=\alpha(Q S)=(\alpha Q) S,
$$

and this is only possible if $\alpha Q=0$. And if there is only one positive vector $\alpha$ (up to a constant multiple) such that $\alpha Q=0$, then we obtain, as above, that $(\alpha P) Q=0$, 
hence $\alpha P=c \alpha$, which assures that $\alpha P=\alpha$. We will see in the next section that this is sometimes an easy way to obtain $\alpha$.

Next we will compute one of the potential operators, $C$, which is defined by

$$
C_{i j}=\lim _{n}\left[N_{j j}^{(n)}-N_{i j}^{(n)}\right] \text {. }
$$

For this we use a formula from [3], namely that

$$
C_{i j}={ }^{j} \hat{\lambda}_{i} \cdot{ }^{i} N_{j j}
$$

where ${ }^{j} \lambda_{i}$ is the limiting probability for hitting $i$ before $j$ in the long run, and $\hat{\lambda}$ is the same quantity for the reverse chain. The former is not easy to compute, but the latter is trivial for a null s.s.ch. Since the reverse chain can move only one step to the left, and since a null chain is "far out" in the long run, we must hit the point further to the right first. Thus if $j>i$, then $C_{i j}=0$, while if $j<i$, then $C_{i j}=$ ${ }^{i} N_{j j}={ }^{i} N_{0 j}$. Thus, for any null s.s.ch.,

$$
C=Q T R \text {. }
$$

For an ergodic (positive recurrent chain) it is more interesting to compute the mean first passage matrix $M$. Then we can obtain $C$, since $C_{i j}=M_{i j} / \alpha_{j}$, for the normalized $\alpha$.

If $i<j$, then $M_{0 j}=M_{0 i}+M_{i j}$, and hence $M_{i j}=m_{j}-m_{i}$. If $i>j$, then we use an identity, from [3],

$$
M_{i j}+M_{j i}={ }^{i} N_{j j} / \alpha_{j}
$$

From this, together with (3.3) and (3.5), and the fact that ${ }^{i} N_{j j}={ }^{i} N_{0 j}$, we obtain

$$
M_{i j}=(Q T R)_{i j} / \alpha_{j}+m_{j}-m_{i}
$$

Since the first term is 0 when $i<j$, this formula is always true.

Next we turn to the dual potential operator $G$, which is also needed for potential theory. Or (equivalently) we will consider the operator $K$, which can take the place of both $C$ and $G$ (see [5]). This is defined as

$$
K_{i j}=\lim _{n}\left[N_{00}^{(n)} \frac{\alpha_{j}}{\alpha_{0}}-N_{i j}^{(n)}\right]=C_{i j}+\lim _{n}\left[N_{00}^{(n)} \frac{\alpha_{j}}{\alpha_{0}}-N_{j j}^{(n)}\right]
$$

The existence of $K$ is necessary and sufficient for the chain to be normal, the condition that enables one to do potential theory. We will show, in the next section, that there are nonnormal s.s.ch. Hence $K$ (or $G$ ) does not always exist, and one cannot expect to express $K$ in terms of $Q$ and $R$, which always exist.

Another way of saying this is to consider ${ }^{j} \lambda_{i}$ : While the limits ${ }^{j} \hat{\lambda}_{i}$ always exist, and are trivial, ${ }^{j} \lambda_{i}$ will not exist for a nonnormal s.s.ch., and hence cannot be obtained from the representation. 
6. Examples. Our first class of examples is the class of random walks, for which Karlin and McGregor obtained a representation in [1]. (Random walks are special s.s.ch., namely those which can take at most one step to the left.) They use a system of polynomials $Q_{i}(x)$ orthogonal with respect to a measure $\psi(x)$ on the interval $[-1,1]$. Their polynomials relate to the matrix $Q$ as follows:

$$
Q_{i}(x)=\sum_{n=0}^{i} Q_{i n} x^{n}
$$

Hence they may be found recursively, as we can determine $Q$. However, then one must still find the measure $\psi$, which is nontrivial; and each component of $P^{n}$ is expressed as an integral. The present representation appears to have the following advantages: (1) If $Q$ is found, $R$ is determined by a trivial matrix inversion. (2) It is sometimes easier to find $R$ and then find $Q=R^{-1}$. (3) Each component of $P^{n}$ is expressed as a finite sum. (4) The representation is applicable to all s.s.ch., not just random walks.

Our next class of s.s.ch. is characterized by the fact that the chain either moves one step to the right, or goes all the way to 0. This is the basic example of [5], and is called a renewal chain in [4] and elsewhere. For these chains $Q$ has the form:

$$
Q_{i i}=1 / \beta_{i} \text { and } Q_{i j}=-\beta_{i-j-1} q_{i-j} / \beta_{i} \text { if } j<i
$$

This can be checked by verifying that $P Q=Q S$.

To express $R$ it is convenient to introduce $x_{n}$ recursively:

$$
x_{0}=1 \text { and } x_{n+1}=\sum_{k=0}^{n} \beta_{n-k} q_{n+1-k} x_{k} .
$$

Then

$$
R_{n j}=x_{n-j} \beta_{j}
$$

Then we find for $P^{n}$,

$$
P_{i j}^{(n+j)}=\frac{\beta_{j}}{\beta_{i}}\left[x_{n+i}-\sum_{k=0}^{i-1} \beta_{i-k-1} q_{i-k} x_{n+k}\right] .
$$

It is interesting to note that the quantity in brackets is independent of $j$.

This class of examples, as well as the next class, illustrates s.s.ch. of the first kind (see [4]). These have the property that the probability of a move to the left, to state $j$, is proportional to a fixed $b_{j}$, and the process is specified by two infinite sets of parameters $\left\{p_{i}\right\}$ and $\left\{b_{j}\right\}$. For renewal chains $b_{j}=0$ for $j>0$. The next class of examples, those called balanced chains in [4], have the vector $b$ proportional to 
the regular measure (where there is one). Again, the parameters $p_{i}$ may be chosen freely. Then $\beta_{i}$ is defined as usual, and we let

$$
\gamma_{i}=\prod_{k=1}^{i}\left(1-\beta_{k}\right)
$$

Balanced chains are characterized by the relation

$$
b_{j}=\beta_{j} / \gamma_{j}
$$

For balanced chains the matrix $Q$ takes on a particularly simple form:

$$
Q_{i i}=1 / \beta_{i} ; \quad Q_{i, i-1}=1-\left(1 / \beta_{i}\right) ; \quad Q_{i j}=0 \text { otherwise. }
$$

And only balanced chains yield a $Q$ of this form. For this simple $Q$ it is easy to find $\alpha$ as the unique solution of $\alpha Q=0$. And if we normalize it by $\alpha_{0}=1$, we verify that $\alpha_{j}=b_{j}$. Inverting $Q$ is also easy:

$$
R_{i j}=\gamma_{i} b_{j}
$$

And thus we obtain

$$
\begin{aligned}
P_{i j}^{(n)} & =\left(1-\beta_{i+n} / \beta_{i}\right) \gamma_{i+n-1} b_{j} & & \text { if } j<i+n, \\
& =\beta_{i+n} / \beta_{i} & & \text { if } j=i+n .
\end{aligned}
$$

Since null chains are normally the hardest ones to represent, we include one specific null chain, to show that the representation may yield simple results even for null chains.

Choose the balanced chain with $p_{i}=i /(i+1)$. Then

$$
\begin{aligned}
P_{i j} & =\frac{1}{(i+1)(i+2)} \quad \text { if } j \leqq i, \\
& =(i+1) /(i+2) \quad \text { if } j=i+1, \quad \beta_{i}=\frac{1}{i+1}, \\
Q_{i i} & =i+1, \quad Q_{i, i-1}=-i, \quad R_{i j}=\frac{1}{i+1} \quad \text { if } j \leqq i . \\
b_{j} & =\alpha_{j}=1 \quad \text { for all } j . \\
C_{i j} & =1+\sum_{k=j+1}^{i} \frac{1}{k} \quad \text { if } i>j . \\
P_{i j}^{(n)} & =\frac{n}{(i+n)(i+n+1)} \quad \text { if } j<i+n, \\
& =(i+1) /(i+n+1) \quad \text { if } j=i+n .
\end{aligned}
$$


The final example is a nonnormal s.s.ch. It will refer back to the nonnormal example of [2]. The latter has two special states, 0 and 1, each the root of a tree with infinitely many branches. From 0 the chain enters a branch of length $n_{2 k-1}$ with probability $(1 / 2)^{k}$, marches deterministically along the branch, and then goes to 1 . From 1 it enters a branch of length $n_{2 k}$ with probability $(1 / 2)^{k}$, marches deterministically to the end, and then goes to 0 . The numbers $n_{k}$ are chosen to be monotone (and very rapidly) increasing. It is then shown that $C_{01}$ and hence ${ }^{1} \hat{\lambda}_{0}$ does not exist. As a matter of fact, the limits in question are not even Abelsummable.

Consider the s.s.ch. which has $P_{i, i+1}=1$ for all $i$, except at the sparsely placed $i=n_{k}$. If $i=n_{2 k-1}$, then $P_{i, i+1}=3 / 4$ and $P_{i 0}=1 / 4$. If $i=n_{2 k}$, then $P_{i, i+1}=2 / 3$, and $P_{i 1}=1 / 3$. Clearly, the chain returns to state 1 infinitely often, hence it is recurrent, and so is the reverse chain $\hat{P}$. This reverse chain marches deterministically till it reaches $1 ; \hat{P}_{10}=1 / 2$ and $\hat{P}_{1, n_{2 k}}=(1 / 2)^{k+1}$, while $\hat{P}_{0, n_{2 k-1}}=(1 / 2)^{k}$. This chain differs from the example described above only in inessential details, and hence the proof of [2] carries over. Therefore $\hat{C}_{01}$ does not exist, and hence neither does ${ }^{1} \lambda_{0}$ or $G_{10}$. Thus $P$ is a s.s.ch. that is not normal.

7. Two applications. As one application of the representation, we will find all eigenvectors of $P$. We are looking for vectors $h$ such that

$$
P h=x h,
$$

and to eliminate constant multiples, we normalize by

$$
h_{0}=1 \text {. }
$$

Let us introduce the special vectors $s^{(x)}$, which are defined by $s_{i}^{(x)}=x^{i}$. Then $s_{0}^{(x)}=1$, and $S s^{(x)}=x s^{(x)}$. And we define $h^{(x)}=Q s^{(x)}$. Then $h^{(x)}$ clearly satisfies (7.2), and

$$
P h^{(x)}=P Q s^{(x)}=Q S s^{(x)}=x Q s^{(x)}=x h^{(x)},
$$

since all the matrices are row-finite. Thus $h^{(x)}$ is one eigenvector for $x$. Conversely, suppose that $h$ satisfies (7.1) and (7.2), then

$$
x R h=R P h=S R h \quad \text { and }(R h)_{0}=1,
$$

but this determines $R h$ as $s^{(x)}$, and hence $h=Q s^{(x)}=h^{(x)}$. Thus $h^{(x)}$ is the unique normalized eigenvector for eigenvalue $x$.

Next let us find out for what values of $x$ the eigenvector is nonnegative. For this it is convenient to find an interpretation for the eigenvector:

$$
h_{i}^{(x)}=\sum_{n=0}^{i} Q_{i n} x^{n}=\frac{1}{\beta_{i}} \chi^{(i)}(x),
$$


where $\chi^{(i)}$ is the characteristic polynomial of ${ }^{i} P$. Since the latter is a finite transient chain, its characteristic polynomial has all of its eigenvalues inside the unit circle, and has a dominant root $r_{i}$, with $0<r_{i}<1$. Thus $\chi^{(i)}(x)>0$ for $x>r_{i}$. In particular, if $x \geqq 1$, then all the characteristic functions are positive, hence $h^{(x)}>0$.

Let $h^{(x)}$ be strictly positive. We then introduce the new transition matrix

$$
P_{i j}^{x}=\frac{P_{i j} h_{j}^{(x)}}{x h_{i}^{(x)}}
$$

which is again a s.s.ch. Choose any $y>x$, and let $h_{i}=h_{i}^{(y)} / h_{i}^{(x)}$. Then $P^{x} h=(y / x) h$, and by the previous result (since $y / x>1$ ), $h>0$. Thus $h^{(y)}>0$.

Therefore, there must be a number $x_{0} \geqq 0$, such that for $x>x_{0}, h^{(x)}>0$, but for $x<x_{0}$ this is never true. Since each component of $h^{(x)}$ is continuous in $x$ (it is a polynomial), we at least have $h^{\left(x_{0}\right)} \geqq 0$. But it follows from (7.1) that $P^{n} h=x^{n} h$. And in a slowly spreading chain $P_{i 0}^{(n)}>0$ for some $n$, hence $h_{i} \geqq\left(1 / x^{n}\right) P_{i 0}^{(n)} h_{0}>0$, if $h$ is nonnegative. Thus each eigenvector which is nonnegative must actually be positive. Thus the only nonnegative solutions of (7.1) and (7.2) are the $h^{(x)}$ with $x \geqq x_{0}$, and these are all strictly positive.

We can further characterize $x_{0}=\sup _{i} r_{i}$ as the sup of the largest eigenvalues, since any $x$ larger than this sup yields a positive $h^{(x)}$, while if $x$ is below the sup, then $x<r_{i}$ for some $i$, and $h^{(x)}>0$ would imply that $h^{\left(r_{i}\right)}>0$, which is false. Thus we have a simple characterization of all the nonnegative eigenvectors of $P$.

In [6] Snell and Lamperti used the Karlin-McGregor representation of random walks to find the Martin boundary of space-time processes formed from random walks. As a second application of the present representation we will extend this to space-time processes formed from s.s.ch. The author is indebted to J. Lamperti for suggesting this application, and for supplying a key step in the proof.

The Martin boundary of a Markov chain consists of all normalized, minimal $\left({ }^{4}\right)$, nonnegative regular functions. This is trivial for a s.s.ch., since our results above imply that the only regular functions are constant. However, the problem becomes interesting if we form the space-time process, i.e., the process whose states are pairs $(i, n)$, where $i$ is a state of the s.s.ch. and $n$ is time. It is customary to restrict this to those pairs $(i, n)$ which can be reached from the starting pair $(0,0)$, which for a s.s.ch. simply means that $n \geqq i$. Thus the space-time process $\bar{P}$ has as states pairs $(i, n)$ with $0 \leqq i \leqq n$, it starts at $(0,0)$, and

$$
\bar{P}(i, n),(j, m)=P_{i j} \quad \text { if } m=n+1,
$$

$$
=0 \quad \text { otherwise }
$$

( ${ }^{4}$ ) A nonnegative regular function $h$ is minimal if whenever $0 \leqq g \leqq h$ for some regular function $g$, then $g=c h$. 
We will find the Martin boundary for such processes under one restriction on $P$ :

$$
P_{i i} \geqq \varepsilon>0 \quad \text { for all } i .
$$

This assumption can be weakened, as will be seen from the proof, but the results found do not hold in complete generality for space-time s.s.ch. (or even for random walks).

Suppose that $h(i, n)$ is a nonnegative regular function for $\bar{P}$. This says that

$$
\sum_{(j, m)} \bar{P}_{(i, n),(j, m)} h(j, m)=h(i, n)
$$

or

$$
\sum_{j} P_{i j} h(j, n+1)=h(i, n) ; \quad \text { and } \quad h(i, n) \geqq 0,
$$

for all $n$, and all $i \leqq n$. Let $g(i, n)=h(i, n+1)$. Then the above equation shows that $g$ is also a regular function, and

$$
h(i, n) \geqq P_{i i} h(i, n+1) \geqq \varepsilon \cdot g(i, n) .
$$

Minimality then requires that $g$ be a constant multiple of $h$, or

$$
h(i, n+1)=\operatorname{ch}(i, n) .
$$

By induction, we then obtain,

$$
h(i, n)=c^{n-i} h(i, i) .
$$

We must distinguish two cases, depending on whether $c=0$. If $c$ does equal 0 , then $h(i, n)=0$ whenever $n>i$. And (7.7) yields $h(i, i)=h(0,0) / \beta_{i}$. Normalization requires $h(0,0)=1$, hence the only possibility for $c=0$ is

$$
h(i, n)=\delta_{i n} / \beta_{i} .
$$

If $c \neq 0$, hence $c>0$, we let $x=1 / c$, and introduce the vector $h$, with $h_{i}=h(i, i) / c^{i}$. Then (7.8) takes the form $h(i, n)=h_{i} / x^{n}$. Normalization requires that $h_{0}=1$, and (7.7) requires that $P h=x h$. Thus we may make use of the results obtained above, concerning eigenvectors. If $c \neq 0$, then

$$
h(i, n)=h_{i}^{(x)} / x^{n}=\left(1 / x^{n}\right)\left[Q s^{(x)}\right]_{i} \text { for some } x \geqq x_{0} .
$$

And the only possible normalized, minimal, nonnegative regular functions are the function (7.9), or the infinite class (7.10). We will show that these are actually all minimal, and hence they form the Martin boundary. It is worth noting that (7.9) is the limit of (7.10) as $x \rightarrow+\infty$, hence the Martin boundary takes the form $\left[x_{0},+\infty\right]$ in terms of the parameter $x$. 
We need only show that all the nonnegative regular functions found above are minimal. A nonnegative regular function $h$ is minimal for $\bar{P}$ if and only if the constant function 1 is minimal for $\bar{P}^{h}$, where

$$
\bar{P}_{(i, n),(j, m)}^{h}=\frac{\bar{P}_{(i, n),(j, m} h(j, m)}{h(i, n)} .
$$

If $h$ is chosen as (7.9), then $\bar{P}^{h}$ marches deterministically from $(i, i)$ to $(i+1, i+1)$. This process has only constants as regular functions, and hence 1 is trivially minimal. Thus the function (7.9) is minimal for $\bar{P}$.

Next let us choose an $x \geqq x_{0}$, and select $h(i, n)=h_{i}^{(x)} / x^{n}$. In (7.11) we always have 0 unless $m=n+1$, and if $m=n+1$ we obtain $P_{i j}^{x}$. Thus $\bar{P}^{n}$ is simply the spacetime process formed from the s.s.ch. $P^{x}$. Thus the question of whether $h$ in (7.10) is minimal for $\bar{P}$ is equivalent to showing that 1 is minimal for the space-time process of $P^{x}$. Our argument will be completed by showing that 1 is minimal for any space-time process formed from a s.s.ch. satisfying (7.6). (Note that if $P$ satisfies this condition, so does $P^{x}$.)

Suppose that we have a space-time process formed from a s.s.ch. satisfying (7.6). Since 1 is a nonnegative, normalized, regular function, the Martin representation theorem guarantees that it can be represented as a convex combination of minimal functions. From (7.6), $P_{i, i+1} \leqq 1-\varepsilon$, hence $\beta_{i} \rightarrow 0$. Thus (7.9) is unbounded, and hence cannot receive positive weight in the representation. Therefore,

$$
1=\int_{x_{0}}^{\infty} \frac{\left[Q s^{(x)}\right]_{i}}{x^{n}} d \mu(x),
$$

where $\mu$ (set of minimal functions) $=1$. But this is of the form

$$
\sum_{k=0}^{i} Q_{i k} \int_{x_{0}}^{\infty} \frac{1}{x^{n-k}} d \mu(x)=1 \quad \text { for all } i \leqq n .
$$

Fix $n$, and let

$$
f_{k}=\int_{x_{0}}^{\infty} \frac{1}{x^{n-k}} d \mu(x), \quad k \leqq n,
$$

then $Q^{(n)} f=1$, where $Q^{(n)}$ is $Q$ restricted to states $0,1, \ldots, n$. Hence $f=R^{(n)} Q^{(n)} f=$ $R^{(n)} 1=1$. Thus

$$
\int_{x_{0}}^{\infty} \frac{1}{x^{m}} d \mu(x)=1 \quad \text { for all } m \geqq 0 .
$$

Using $m=0,1,2$,

$$
1=\int_{x_{0}}^{\infty} 1 \cdot \frac{1}{x} d \mu(x) \leqq\left(\int_{x_{0}}^{\infty} 1 d \mu(x) \int_{x_{0}}^{\infty} \frac{1}{x^{2}} d \mu(x)\right)^{1 / 2}=1,
$$

and we have equality in Schwartz's inequality, which implies that $1 / x$ is proportional to 1 a.e. Hence $x=1$ a.e.; that is, $\mu(\{1\})=1$. Thus 1 is a minimal function. 
8. Extensions of the results. First of all we will consider duals of s.s.ch. For these we need a measure $\alpha>0$ which is superregular, and is chosen to be regular if such a measure exists. Then the dual chain has transition matrix

$$
\hat{P}_{i j}=\alpha_{j} P_{j i} / \alpha_{i} \text {. }
$$

In particular, if the chain is recurrent, $\alpha$ is unique up to a constant multiple, and hence $\hat{P}$ is uniquely determined. It is convenient to introduce the diagonal matrix $D$, with $D_{i i}=1 / \alpha_{i}$. Then (8.1) may be written in matrix form as

$$
\hat{P}=D P^{T} D^{-1}
$$

This immediately yields a representation for dual-s.s.ch., namely $\hat{P}=D R^{T} S^{T} Q^{T} D^{-1}$, or

$$
\hat{P}=\hat{Q} S^{T} \hat{R}, \quad \text { where } \quad \hat{Q}=D R^{T} \quad \text { and } \quad \hat{R}=Q^{T} D^{-1}
$$

All the dual matrices are column-finite, and

$$
\hat{P}^{n}=\hat{Q}\left(S^{T}\right)^{n} \hat{R}
$$

expresses an entry in the $n$th power of the transition matrix as a finite sum.

The duals of $Q$ and $R$ are upper-triangular, and inverses of each other in the strong sense. Instead of having row-sums equal to 1 , we have the relations

$$
\alpha \hat{Q}=1^{T} \quad \text { and } \quad 1^{T} \hat{R}=\alpha .
$$

For instance, for the null balanced chain given in $\S 6$ we have $\alpha_{i}=1$, hence $\hat{P}=P^{T}, \hat{Q}=Q^{T}$, and $\hat{R}=R^{T}$. This dual chain is again an interesting process. Moving to the right it behaves like an independent trials process, but it can only move one step to the left.

There are certain s.s.ch. whose duals are also s.s.ch. In this case we obtain two representations for it, (1.1) and (8.3). Such chains are characterized by the fact that they can move only one step to the right, and only one to the left. These chains are precisely the random walks. But random walks have the property that there always is a unique regular measure $\alpha$, and the chain is "reversible," i.e., $\hat{P}=P$. Therefore, $Q S R=\hat{Q} S^{T} \hat{R}$.

Next we consider the class of $k$-spreading chains. These have the natural numbers as states, have states communicating, are restricted to take no more than $k$ steps to the right, and $P_{i, i+k}>0$ for every $i$. In particular, for $k=1$ we obtain the s.s.ch. We obtain a representation for $k$-spreading chains in almost the same manner as for s.s.ch.

We define the rows of $R$ inductively. For $n<k, R_{n j}=\delta_{n j}$. And row $n+k$ is the $n$th row of $R$ times $P$. This gives us an interpretation of $R_{m j}:$ If $m=q k+r$, then 
$R_{m j}=P_{r j}^{(q)}$. The matrix $R$ is again nonnegative and triangular, and we obtain $Q$ as its inverse. By definition, $R P^{n}=S^{n k} R$, and hence

$$
P^{n}=Q\left(S^{k}\right)^{n} R
$$

Thus our representation is complicated only by having $S^{k}$ in place of $S$.

It is not only that there are many more $k$-spreading chains than s.s.ch. that makes them interesting. It is the fact that many types of chains studied in the literature can be considered as $k$-spreading if we renumber the states. For example, sums of independent random variables, where each variable takes on only a finite number of values, can be reconstructed as a $k$-spreading chain. If $X_{n}=S_{1}+\cdots$ $+S_{n}$, and the largest and smallest values taken on by $S_{i}$ are $+a$ and $-b$, respectively, then we can renumber the states to obtain an $(a+b)$-spreading chain.

We will illustrate this for the cases where $S_{i}$ takes on the values $-1,0,+1$, and +2 . We number the states in the following order:

$$
0,1,-1,2,3,-2,4,5,-3,6,7,-4, \ldots
$$

and we have a 3-spreading chain.

There is another rich class of examples which may be obtained from our representation. Consider any s.s.ch. $P$, and watch it by means of a random clock. Suppose that the clock goes off at integer time $n$ with probability $a_{n}$, and we watch the original chain only when the clock goes off. Let us introduce $f(t)$, the generating function of the random times:

$$
f(t)=\sum_{n=0}^{\infty} a_{n} t^{n}
$$

The transition matrix of the new process is $f(P)$, hence it is represented by

$$
f(P)=Q \cdot f(S) \cdot R
$$

While $f(S)$ is more complicated than $S$, it is the transition matrix of a sums-ofindependent random variables ladder process (it can move only to the right). Hence, if we can compute the $n$th power of the generating function, we can compute the $n$-step transition probabilities. It is worth noting that $f(S)$ has $\left(a_{0}, a_{1}\right.$, $\left.a_{2}, \ldots\right)$ as its first row, and each following row is shifted right by 1 .

Finally, let us see what the representation says for finite s.s.ch.; i.e., for finite chains, with states $0,1, \ldots, n$, which can move from state $i<n$ only one step to the right. We may still introduce the matrix $R$ inductively, as before. It is triangular, and hence it has an inverse $Q$. But it is no longer true that $R P=S R$, since this relation breaks down in the last row. The $n$th row of $R$ is the 0 th row of $P^{n}$; when this is multiplied by $P$, we obtain the 0 th row of $P^{n+1}$. But $n+1$ is the order of the 
matrix, hence $P^{n+1}$ is expressible as a linear combination of the smaller powers. Hence

$$
R P=\bar{S} R ; \quad \bar{S}=\left(\begin{array}{ccccc}
0 & 1 & 0 & 0 & \cdots \\
0 & 0 & 1 & 0 & \cdots \\
0 & 0 & 0 & 1 & \cdots \\
\cdot & \cdot & \cdot & \cdot & \cdot \\
c_{0} & c_{1} & c_{2} & \cdots & c_{n}
\end{array}\right) ; \quad P=Q \bar{S} R .
$$

I am indebted to $\mathrm{H}$. Mirkil for pointing out that this is the rational normal form of the finite matrix $P$. It is of mathematical interest because it represents $P$ within the field which contains its components-unlike the Jordan form. Thus our representation leads to an interesting canonical form even for finite s.s.ch. It also motivates us to point out that the representation has the property, in general, that $Q$ and $R$ are real, and if $P$ has components which are rational then so will $Q$ and $R$.

\section{REFERENCES}

1. S. Karlin and J. McGregor, Random walks, Illinois J. Math. (1) 3 (1959), 66-81.

2. J. G. Kemeny and J. L. Snell, Notes on discrete potential theory, J. Math. Anal. Appl. (1) 3 (1961), 117-121.

3. - Potentials for denumerable Markov chains, J. Math. Anal. Appl. (2) 3 (1961), 196-260.

4. J. G. Kemeny, Slowly spreading chains of the first kind, J. Math. Anal. Appl. (to appear).

5. J. G. Kemeny, J. L. Snell and A. W. Knapp, Denumerable Markov chains, Van Nostrand, New York, 1966.

6. J. Lamperti and J. L. Snell, Martin boundaries for certain Markov chains, J. Math. Soc. Japan (2) 15 (1963), 113-128.

Dartmouth College, HANOVER, New HAMPSHIRE 